\title{
Urban Electromagnetic Spider Web
}

\author{
Min Jiang \\ Ningbo Polytechnic, Ningbo, China \\ Email: chiangm@163.com
}

How to cite this paper: Jiang, M. (2017) Urban Electromagnetic Spider Web. Open Access Library Journal, 4: e4007. https://doi.org/10.4236/oalib.1104007

Received: October 9, 2017

Accepted: November 19, 2017

Published: November 22, 2017

Copyright $\odot 2017$ by author and Open Access Library Inc.

This work is licensed under the Creative Commons Attribution International License (CC BY 4.0).

http://creativecommons.org/licenses/by/4.0/

\section{(c) (i) Open Access}

\begin{abstract}
Here design of electromagnetic spider webs imitating the actual spider web and trigram cropland structure, using the principle of bionics will combine Telluric Current, electricity, Atmospheric electronic, E-compass, 4G-GSM wave Switch, low and high frequency wireless transmitting and receiving devices. WIFI devices Switch. In theory, the electromagnetic spider webs induction method in Maxwell equation is adopted, and simple instruments and equipment are made by combining some software, such as wavelet transform, Mat-Lab simulation and configuration software. At ordinary times, all kinds of abnormal electromagnetic signals are detected at any time before the earthquake. In war, device can be used to detect all kinds of flying objects, such as stealth aircraft and cruise missiles, through the spider webs. Low equipment costs, reliable operation, we have forty years of wisdom crystallization [1].
\end{abstract}

\section{Subject Areas}

Environmental Sciences

\section{Keywords}

Earthquake Prediction, Magnetic Switch Module, Wireless Transmitting and Receiving Module, GPRS Network, WIFI Switch Nets, Electromagnetic Spider Web, Telluric Electricity

\section{The Urban Electromagnetic Spider Web Structure}

The urban area of Ningbo is about 1000 square kilometers. Using $4 \mathrm{G}$ network of Electromagnetic Spider Web's structure shown in Figure 1(a) and Figure 1(b). Usually, The wireless transmission and WIFI module placed in the areas where earthquakes are usually from a distance of 30 meters, 300 meters, $3 \mathrm{KM}, 30 \mathrm{KM}$ four groups, the super heterodyne receiving device corresponding received signals by using the host, "land" and the electronic compass were placed in four groups, to know the earth electromagnetic anomaly. Remote monitoring based 

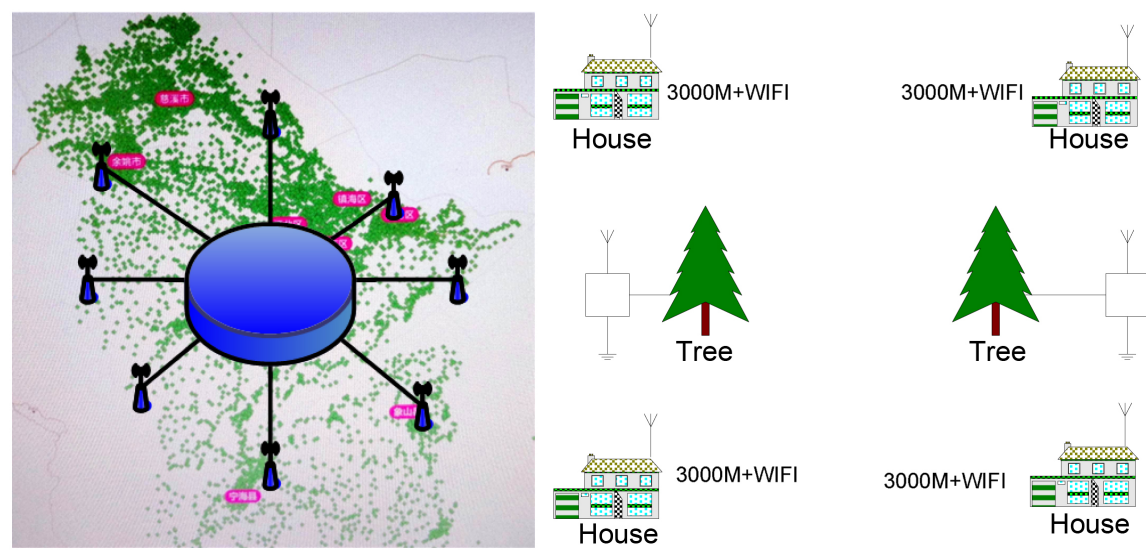

Figure 1. (a) The $4 \mathrm{G}$ network of urban of Ningbo electromagnetic spider web diagram. (b) Every point connection diagram.

on GPRS network and WIFI technology can measure local electromagnetic field changes and warn early in a few days before large earthquakes, and analyze the location of earthquake epicenter. The use of electromagnetic spider nets is a kind of surface sensor, with three-dimensional detection method to determine the three elements of the earthquake, in theory, then point, line detection is more advanced. The received signals are analyzed by wavelet transform and MATLAB simulation, and the mathematical statistics analysis of multi-channel signals is used. If you want to know the location of the epicenter, you have to put a number of electromagnetic spider webs. Preliminary experiments have reached the basic design requirements. After the discovery of electromagnetic anomalies, use special timer to start the device 6 times per hour, and wait for 3 minutes each time to wait for the remote control signal [2].

As the number of electromagnetic spider webs user's increases, there are only a few AP devices, without rewiring. While the global unified WIFI standard makes it different from the cellular carrier technology, the same Wi-Fi user can use WLAN services in various countries of the world. Outdoor equipment, with low cost and low power consumption, is a promising method for short impending earthquake prediction. Use wireless and WIFI control transmitter. Through the special wavelet analysis and judgment, we can accurately determine the epicenter location of the earthquake. This method can be used to determine the range of electromagnetic anomalies, to determine the type of earthquake and the location of the epicenter. The self-made equipment has low cost and high reliability. It can be used to detect the change of real-time electromagnetic field. This device is in all $4 \mathrm{G}$ networks in the world. In normal electromagnetic time, the remote control alarm circuit does not start the GSM or Wi-Fi switch, and when the abnormal electromagnetic is generated, the remote automatic alarm is made, and after the alarm is locked, the remote controller can release the remote controller to restore the original state as long as the OFF is issued remotely. GSM switch or Wi-Fi switch power supply can charge more than 200 times at one time. It is very convenient. At ordinary times, the electromagnetic spider net scans four times per minute, and each 
time it turns on 5 - 10 seconds, the electric field and magnetic field are detected. Combined with the GPRS remote monitoring device or Wi-Fi switch, the location of the epicenter of the earthquake can be easily determined, and the accurate analysis of the three elements of the earthquake [3].

\section{Gauss Flux Theorem Experimental Circuit}

The electric charge classified as "free charge"-for example, the charge that transferred in static electricity or the charge on a capacitor plate. In contrast, "bound charge" arises only in the context of dielectric (polarizable) materials. (All materials are polarizable to some extent.) When such materials placed in an external electric field, the electrons remain bound to their respective atoms, but shift a microscopic distance in response to the field, so that they are more on one side of the atom than the other is. (Figures 2(a)-(c)) All these microscopic displacements add up to give a macroscopic net charge distribution, and this constitutes the "bound charge" [4].

It pointed that the net magnetic flux passing through either closed surface equals zero, i.e., the flux passing through is equal to the flux passing through, and its algebraic sum is zero, B is the flux density, and $S$ is any closed surface. This formula shows that the lines of force are continuous, and are closed lines that do not have an initial end or a terminal. These lines of force are closed curves [5].

Telluric electricity is in a few meters or farther from the underground, buried two lead, then the line ends connected to the ammeter or voltage meter, to observe the change of the change of the pointer be directly observed on the earth's natural electromagnetic field. "Telluric electricity power" is like the pressure of the earth. Figure 3(b) is a simple "telluric electricity power" where an electronic spider web placed.

The E-compass uses the special data processing method to improve the accuracy of the system. because the system uses the anti-interference technology, thereby reducing the influence caused by other factors, the system accuracy is further improved, in addition, the system itself can measure the earth magnetic anomaly, so it can be easily composed of 3 dimensional measurement system with another the general magnetic field sensor, to eliminate the phenomenon of tilt. As the system works implemented by various processing platforms, it has good portability, can be widely used in positioning systems, and has good reliability and high accuracy. Figure 3 (a) and Figure $3(\mathrm{~b})$ are the electronic compass experimental circuit [6].

\section{Faraday Law of Electromagnetic Induction Experimental Circuit}

It is an electronic compass device in an electromagnetic spider web. Typically, the $3 \mathrm{D}$ electronic compass consists of Hall magneto resistive sensors, ferromagnetic devices, sensors, amplifier circuits, and microcontroller MCU. The 3D 


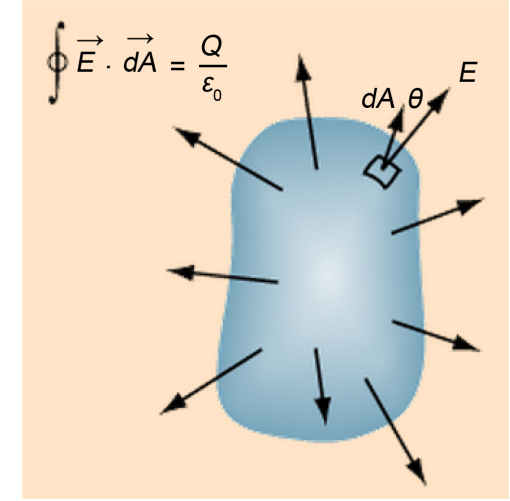

(a)

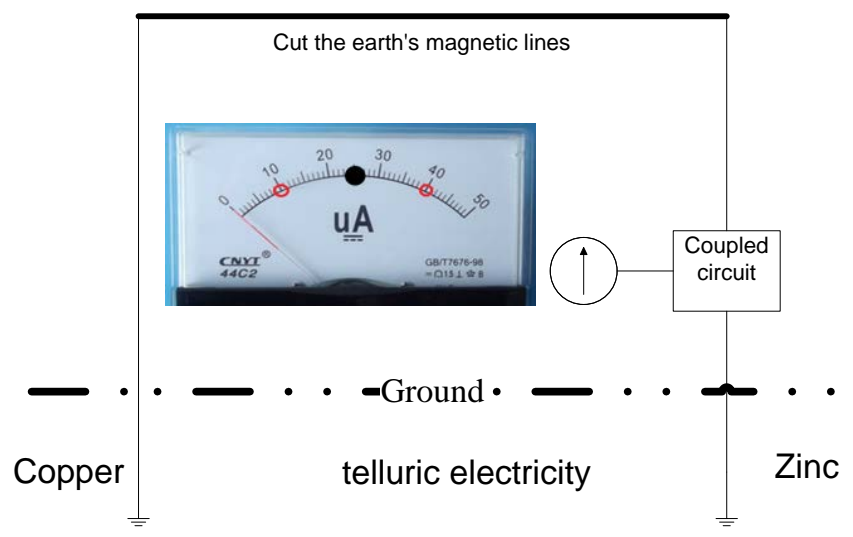

(b)

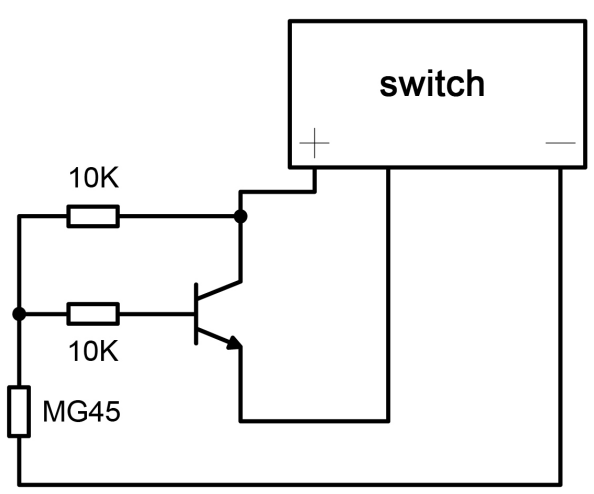

(c)

Figure 2. (a) Gauss flux theorem diagrammatic sketch. (b) Gauss flux theorem experimental circuit. (c) Gauss flux theorem experimental circuit0, 1 switch circuit.

magnetic field sensor used to measure the earth's magnetic field, ordinary compass tilt sensor in equipment for compensation in non-plane position of abnormal state; MCU soft hardware processing equipment and the tilt sensor signal and a data output circuit using the hard iron compensation. The electronic compass uses a three-dimensional magnetic field sensor that facilitates the adjustment of sensors on each dimension to detect the intensity of the earth's magnetic field in that direction. The magnetic declination at the same location is 
DN6851

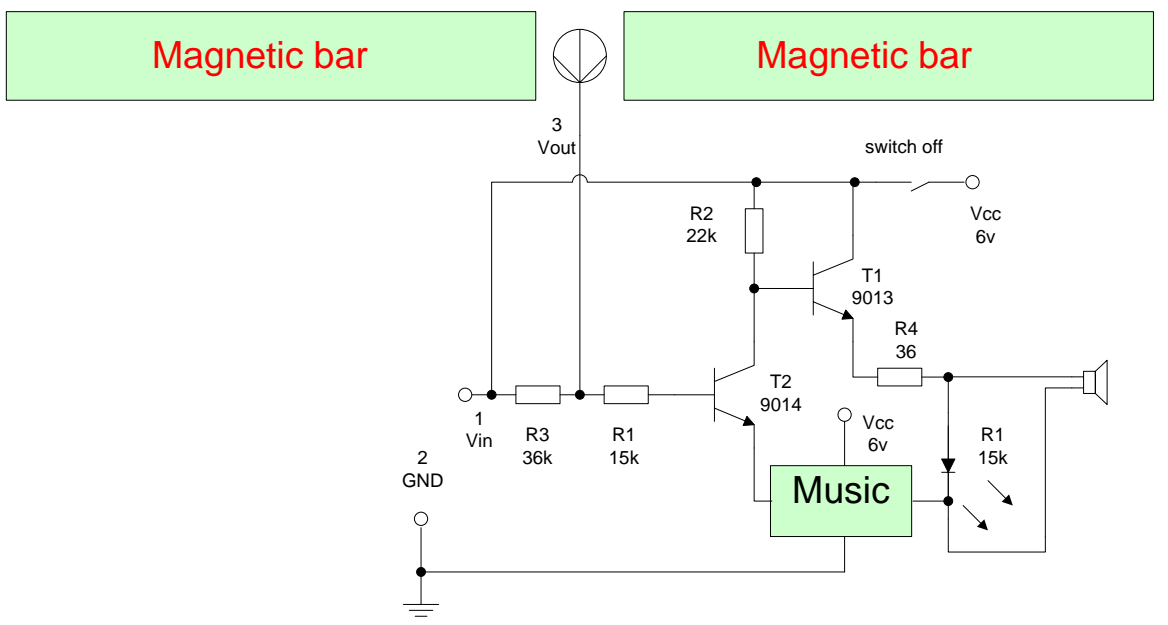

(a)

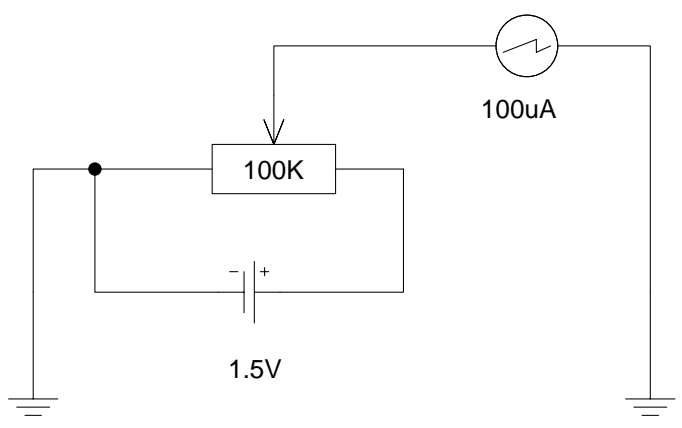

(b)

Figure 3. (a) The electronic compass experimental circuit. (b) The telluric electricity experimental circuit.

not a fixed value, the determination of the declination of the day eventually there was a small difference, with long-term changes and characteristics of magnetic declination change on Sunday. Effect of meteorological conditions on the declination is also great, especially in the lightning weather not observed. The environmental impact of significant geomagnetic declination, due to the magnetic properties of the magnetic meridian, the situation is more complex, and are not parallel to each other, the line is longer, the greater the error. The initial use should be properly magnetized magnet. Figures 4(a)-(c) are Maxwell Equations 3 Faraday electromagnetic induction law Simple compass experimental circuit.

\section{Atmospheric Electric Field Experimental Circuit}

Electromagnetic spider webs can only count number of airplanes shadow on the ground the number of echoes is consistent with the radar high frequency statistics. Atmospheric electric field: a physical field that exists in the atmosphere and interacts with electrically charged substances. In a clear electric field, the electric field in the horizontal direction neglected. In the field of atmospheric electricity, regulation to vertical downward called atmospheric electric field, the gradient of 


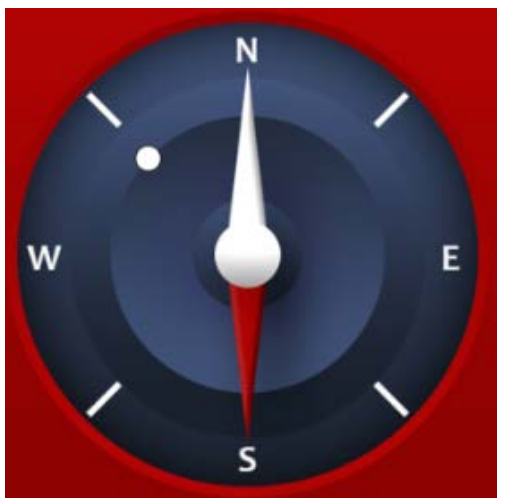

(a)

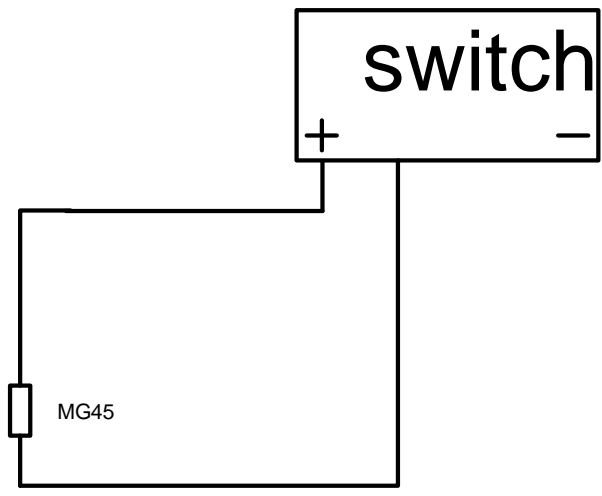

(b)

Integral form

$\oint \vec{B} \cdot d \vec{A}=0$

Differential form

$$
\nabla \cdot B=0
$$

(c)

Figure 4. (a) Simple compass experimental circuit. (b) 0, 1 switch circuit. (c) Maxwell Equations 3 Faraday electromagnetic induction law.

the potential gradient. The electric field in sunny weather increases with latitude, which called latitude effect. In industrial areas, the strength of the field increases to hundreds of volts per meter due to high concentrations of aerosols in the air. The electric field of sunny day has two periodic changes, namely, day and year. The antenna and equipment easily installed on the monitoring point of the $4 \mathrm{G}$ network. Figures 5(a)-(c) is Maxwell's equations four: Ampere's loop theorem [7].

\section{Wi-Fi Switch Device}

Intelligent switch is a unit that uses the combination and programming of control board and electronic component to realize the intelligent switch control of circuit. Switching control also called on and off control. The conventional switch control is difficult to meet the requirement of further improving the control accuracy and energy saving. This is based on people's knowledge and experience, and according to the change of the controlled object of the actual error (or process) inertia, pure lag and disturbance, according to a certain mode selection switch of different control strategies for control called intelligent switch control.

\section{Summary}

The design plan can detect all kinds of abnormal electromagnetic signals at any time before the earthquake. Low equipment costs, reliable operation, we have forty years of wisdom crystallization. Its circuit control system is simple. Can foreknow, can successfully find the electromagnetic field and electromagnetic wave anomaly alarm method, and the formation of a comprehensive global 


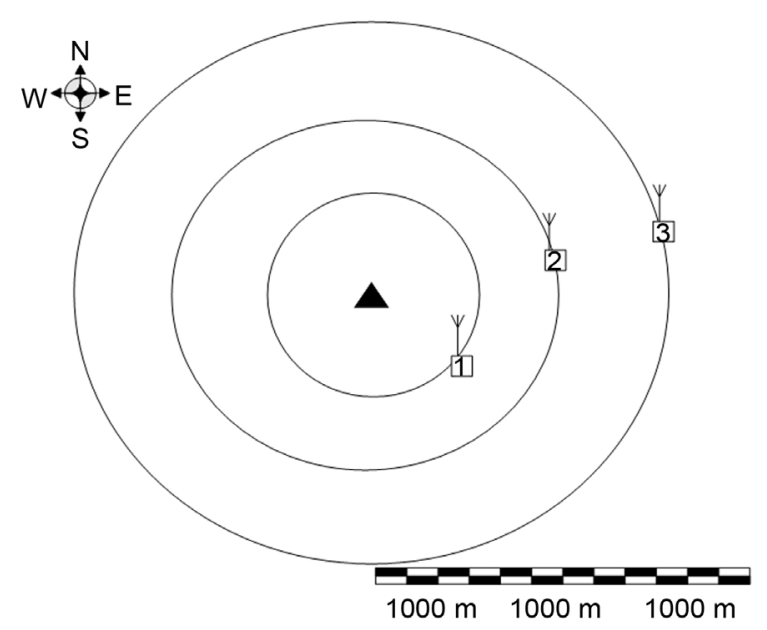

(a)

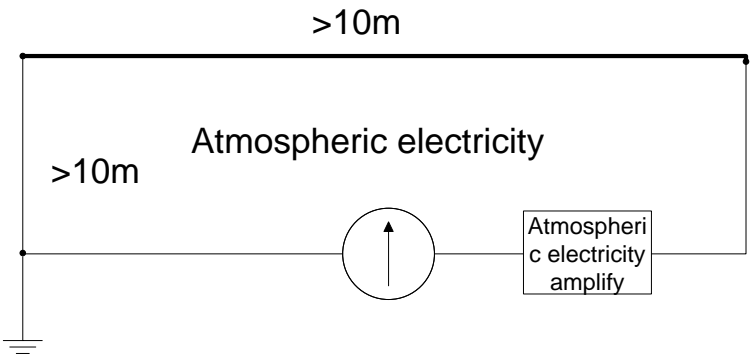

(b)

$$
\left\{\begin{array}{l}
\oiint_{S} \mathbf{E} \cdot \mathrm{d} \mathbf{S}=\frac{1}{\varepsilon_{0}} q \\
\oiint_{S} \mathbf{B} \cdot \mathrm{d} \mathbf{S}=0 \\
\oint_{L} \mathbf{E} \cdot \mathrm{d} \mathbf{l}=\iint_{S} \frac{\partial \mathbf{B}}{\partial t} \cdot \mathrm{d} \mathbf{S} \\
\oint_{L} \mathbf{B} \cdot \mathrm{d} \mathbf{l}=\mu_{0} I+\mu_{0} \varepsilon_{0} \iint_{S} \frac{\partial \mathbf{E}}{\partial t} \cdot \mathrm{d} \mathbf{S}
\end{array}\right.
$$

(c)

Figure 5. (a) Three point connection diagram. (b) One of the Maxwell's equations experimental circuits. (c) Ampere's loop theorem.

monitoring system, and will promote the emergence and development of earthquake prediction in seismology and earthquake prediction. "I hope your generation can solve earthquake prediction." on the earthquake prediction Premier Zhou's many instructions and speech let me deeply feel swept down irresistibly from a commanding height. The unity is put forward in this paper. To integrate the whole city into the study of electromagnetic spider web, we implemented a practical four-way electromagnetic spider web design, hoping to protect the safety of human life and property in earthquake prediction.

\section{References}

[1] Jiang, M. (1992) Multi Point Timing Control Circuit. Electric World, No. 4, 42. 
[2] Jiang, M. (1996) Sound and Light Indicator of Electronic Compass. Electric World, No. 2, 33 .

[3] Jiang, M. (2010) Earthquake Prediction Micro Integrated Measurement Recorder. Technology Innovation Herald, No. 29, 22-23.

[4] Jiang, M. (2013) How to Weave the Electromagnetic Spider Web to Predict Earthquakes. 2013 3rd International Conference on Education and Education Management (EEM 2013), Singapore, 15 November 2013, 546-551.

[5] Jiang, M. (2014) Electromagnetic Spider Web Application in Earthquake Prediction. International Core Journal of Scientific Research \& Engineering Index, 539, 111-114. https://doi.org/10.4028/www.scientific.net/AMM.539.101

[6] Jiang, M. (2014) The Group of Electromagnetic Spider Web, Economic. Business Management and Education Innovation (EBMEI 2014), Singapore, 5-6 November 2014, 287-291.

[7] Jiang, M. (2017) Three-Point Electromagnetic Spider Web. Open Access Library Journal, 4, e3582 https://doi.org/10.4236/oalib.1103582

Submit or recommend next manuscript to OALib Journal and we will provide best service for you:

- Publication frequency: Monthly

- 9 subject areas of science, technology and medicine

- Fair and rigorous peer-review system

- Fast publication process

- Article promotion in various social networking sites (LinkedIn, Facebook, Twitter, etc.)

- Maximum dissemination of your research work

Submit Your Paper Online: Click Here to Submit

Or Contact service@oalib.com 